\title{
Front Matter: Volume 9951
}

, "Front Matter: Volume 9951," Proc. SPIE 9951, Optical System Alignment, Tolerancing, and Verification X, 995101 (23 November 2016); doi:

$10.1117 / 12.2263073$

SPIE Event: SPIE Optical Engineering + Applications, 2016, San Diego, California, SPIE. United States 


\section{PROCEEDINGS OF SPIE}

\section{Optical System Alignment, Tolerancing, and Verification $X$}

José Sasián

Richard N. Youngworth

Editors

28-29 August 2016

San Diego, California, United States

Sponsored and Published by

SPIE

Volume 9951

Proceedings of SPIE 0277-786X, V. 9951

SPIE is an international society advancing an interdisciplinary approach to the science and application of light.

Optical System Alignment, Tolerancing, and Verification X, edited by José Sasián,

Richard N. Youngworth, Proc. of SPIE Vol. 9951, 995101 - (c) 2016 SPIE

CCC code: $0277-786 X / 16 / \$ 18 \cdot$ doi: $10.1117 / 12.2263073$

Proc. of SPIE Vol. $9951995101-1$

Downloaded From: https://www.spiedigitallibrary.org/conference-proceedings-of-spie on 26 Apr 2023 Terms of Use: https://www.spiedigitallibrary.org/terms-of-use 
The papers in this volume were part of the technical conference cited on the cover and title page. Papers were selected and subject to review by the editors and conference program committee. Some conference presentations may not be available for publication. Additional papers and presentation recordings may be available online in the SPIE Digital Library at SPIEDigitallibrary.org.

The papers reflect the work and thoughts of the authors and are published herein as submitted. The publisher is not responsible for the validity of the information or for any outcomes resulting from reliance thereon.

Please use the following format to cite material from these proceedings:

Author(s), "Title of Paper," in Optical System Alignment, Tolerancing, and Verification $X$, edited by José Sasián, Richard N. Youngworth, Proceedings of SPIE Vol. 9951 (SPIE, Bellingham, WA, 2016) Sixdigit Article CID Number.

ISSN: 0277-786X

ISSN: 9781510602946 (electronic)

ISBN: 9781510602939

ISBN: 9781510602946 (electronic)

Published by

SPIE

P.O. Box 10, Bellingham, Washington 98227-0010 USA

Telephone +1 3606763290 (Pacific Time) · Fax +1 3606471445

SPIE.org

Copyright (C) 2016, Society of Photo-Optical Instrumentation Engineers.

Copying of material in this book for internal or personal use, or for the internal or personal use of specific clients, beyond the fair use provisions granted by the U.S. Copyright Law is authorized by SPIE subject to payment of copying fees. The Transactional Reporting Service base fee for this volume is $\$ 18.00$ per article (or portion thereof), which should be paid directly to the Copyright Clearance Center (CCC), 222 Rosewood Drive, Danvers, MA 01923. Payment may also be made electronically through CCC Online at copyright.com. Other copying for republication, resale, advertising or promotion, or any form of systematic or multiple reproduction of any material in this book is prohibited except with permission in writing from the publisher. The CCC fee code is 0277-786X/16/\$18.00.

Printed in the United States of America.

Publication of record for individual papers is online in the SPIE Digital Library.

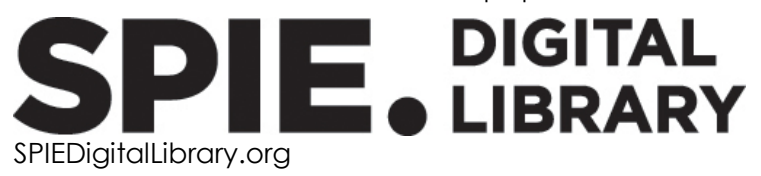

Paper Numbering: Proceedings of SPIE follow an e-First publication model. A unique citation identifier (CID) number is assigned to each article at the time of publication. Utilization of CIDs allows articles to be fully citable as soon as they are published online, and connects the same identifier to all online and print versions of the publication. SPIE uses a six-digit CID article numbering system structured as follows:

- The first four digits correspond to the SPIE volume number.

- The last two digits indicate publication order within the volume using a Base 36 numbering system employing both numerals and letters. These two-number sets start with $00,01,02,03,04$, 05, 06, 07, 08, 09, OA, OB ... OZ, followed by 10-1Z, 20-2Z, etc. The CID Number appears on each page of the manuscript. 


\title{
Contents
}

\author{
$\checkmark \quad$ Authors \\ vii Conference Committee \\ ix Introduction \\ xi The advanced LIGO detectors in the era of first discoveries (Plenary Paper) [9960-200]
}

\section{SESSION 1 SPACE SYSTEMS AND JWST ALIGNMENT AND INTEGRATION I}

995102 Multispectral optical telescope alignment testing for a cryogenic space environment [9951-1]

995103 Tolerancing, alignment and test of the Transiting Exoplanet Survey Satellite (TESS) optical assembly [9951-2]

995104 Data veracity checks for the alignment of the JWST optical telescope element [9951-3]

995105 Optical testing and verification methods for the James Webb Space Telescope Integrated Science Instrument Module element [9951-4]

995106 Critical science instrument alignment of the James Webb Space Telescope (JWST) Integrated Science Instrument Module (ISIM) [9951-5]

\section{SESSION 2 ALIGNMENT METHODS AND ALGORITHMS}

995107 High precision optomechanical assembly using threads as mechanical reference [9951-6]

995108 A toolbox of metrology-based techniques for optical system alignment (Invited Paper) [9951-7]

995109 Design and performance of a new compact adaptable autostigmatic alignment tool [9951-8]

9951 OA Array imaging system for lithography [9951-9]

SESSION 3 SPACE SYSTEMS AND JWST ALIGNMENT AND INTEGRATION II

9951 OC Alignment of the James Webb Space Telescope Integrated Science Instrument Module Element [9951-11]

9951 OD JWST science instrument pupil alignment measurements [9951-12] 
9951 OE JWST's optical telescope simulator for verification of the Integrated Science Instrument Module [9951-13]

9951 OF How to align a new detector and micro shutter inside JWST's Near Infrared Spectrograph (NIRSpec) [9951-14]

9951 OG Mechanical blind gap measurement tool for alignment of the JWST Optical Telescope Element [9951-15]

\section{SESSION 4 ASPHERES, TOLERANCING, AND VERIFICATION}

$9951 \mathrm{OH} \quad$ Precision spacers as compensators [9951-16]

9951 ol Asphere wedge and decenter: What you see is not always what you get [9951-17]

9951 0J The structure function as a metric for roughness and figure [9951-18]

9951 OK Measurement of the alignment of the surfaces and the edges of aspheric lenses [9951-19]

9951 OL Method for quick thermal tolerancing of optical systems [9951-20]

9951 OM Sensitivity analysis in the test of a parabolic trough solar collector (PTSC) with flat nullscreens [9951-21]

MONDAY POSTER SESSION

9951 ON Cryogenic optical test planning using the Optical Telescope Element Simulator with the James Webb Space Telescope Integrated Science Instrument Module [9951-23]

995100 Toward image registration process: Using different interpolation methods in case of subpixel displacement [9951-24]

9951 OP Analysis of the influence of the aperture size on the differences of $L{ }^{*} a{ }^{*} b$ chromatic coordinates in a spectrocolorimeter [9951-25]

WEDNESDAY POSTER SESSION

$99510 Q \quad$ Using short helically wrapped single-mode fibers as illuminated fiducials [9951-22]

iv 


\section{Authors}

Numbers in the index correspond to the last two digits of the six-digit citation identifier (CID) article numbering system used in Proceedings of SPIE. The first four digits reflect the volume number. Base 36 numbering is employed for the last two digits and indicates the order of articles within the volume. Numbers start with 00, 01, 02, 03, 04, 05, 06, 07, 08, 09, 0A, 0B...0Z, followed by 10-1Z, 20-2Z, etc.

Alves de Oliveira, Catarina, ON Andre, James, 03 Antonille, Scott R., 05, 0C, OD, ON Aronstein, David L., 05, OC Balderas-Mata, S. E., OP Balonek, Gregory, 03 Bartoszyk, Andrew E., 05, 0C Beaton, Alexander, OD Bergeron, Guy, 07

Berrier, Josh, OC

Beutler, Andreas, OK Birkmann, Stephan, ON Blake, Peter N., 08 Bond, Nicholas A., ON Bos, Brent J., 08

Bowers, Charles W., 05

Campos-García, Manuel, OM

Cantin, Mario, 07

Chambers, Victor J., 08

Champagne, James, 02

Chang, William S., OE

Chesbrough, Christian, 03

Chrisp, Michael, 03

Chu, Jenny, OE

Clark, Kristin, 03

Cofie, Emmanuel, 05, 0C

Collins, Nicholas R., 05

Comber, Brian J., 05

Coulter, Phillip, 08, OC, OD

Davis, Clinton R., OE, ON

Desnoyers, Nichola, 07

Díaz-Uribe, José Rufino, OM

Dixon, William V., ON

Edelstein, Jerry, $0 Q$

Eder, Robert, OF

Ehrenwinkler, Ralf, OF

Eichhorn, William L., 05, 08, 0C, OE

Farey, Mike, 04

Flores Padilla, Deyanira, 00

Flores, Jorge L., OP

Fuchs, U., Ol

Glasse, Alistair C., 05

Glassman, Tiffany, 04

Gracey, Renee M., 05, 06, 0C

Greeley, Bradford W., OE, ON

Gum, Jeffrey S., 08

Gunn, Chris, OF

Hadjimichael, Theodore J., 08, 0C

Hagopian, John G., 08
Hartig, George F., 05, OC, OD, OE

Hayden, Joseph E., 08, OC

Hetherington, Samuel E., 08

Hooser, Preston, 02

Howard, Joseph M., 05, 06, 0C

Huerta-Carranza, Oliver, OM

Hylan, Jason, $\mathrm{OC}$

Ishikawa, Yuzo, OQ

Jensen, Peter, OF

Jimenez-Hernández, Hugo, 00

Johnson, Thomas E., OF

Kehoe, Michael, $\mathrm{OH}$

Kelly, Douglas M., 05, OD

Kimble, Randy A., 05, OE

Kiontke, S. R., Ol

Kirk, Jeffrey R., 05, OE

Kirner, Raoul, OA

Kreischer, Cody, $\mathrm{OH}$

Kubalak, David A., 05, 06, 08, OC, OD, OE

Kuhn, William P., 09

Lamontagne, Frédéric, 07

Landsman, Wayne B., 05

Lee, David, OD

Levi, Josh, 04

Liepmann, Till, 04, 0G

Lindler, Don J., 05

MacDonald, Robert, 03

Malaurie, Pauline, $0 \mathrm{~A}$

Malumuth, Eliot M., 05, ON

Martel, André R., ON

Maszkiewicz, Michael, 05, OD

Mclean, Kyle F., 08, 0C

McMann, Joseph C., 08

Medina-Marquez, J., OP

Melendez, Marcio, ON

Merkle, Frank, OF

Miskey, Cherie L., 05, OC, ON

Moreno-Oliva, Víctor Iván, OM

Mott, Brent, OF

Mueller, Kevin, OA

Newswander, Trent, 02

Noell, Wilfried, OA

Ohl, Raymond G., 05, 08, OC, OD, OE, ON

Parks, Robert E., OJ

Peña-Conzueloa, Andrés, OM

Poppett, Claire, $0 Q$

Primeau, Brian, 03

Redman, Kevin W., 08, 0C

Reichard, Timothy A., ON 
Reynosa Canseco, Jaqueline, 00

Rieke, Marcia J., 05

Roedel, Andreas, OF

Rohrbach, Scott O., 05, 06, 0C

Rowlands, Neil, 05

Rumler, Peter, OF

Sabatke, Derek S., 05, 06, OC, OE, ON

Sampler, Henry P., 08

Santiago-Alvarado, Agustín, OM

Scharf, Toralf, OA

Schweiger, Paul, OD

Shiri, Ron, ON

Sigg, Daniel, xi

Silber, Joseph H., OQ

Smith, Corbett T., 05

Smith, J. Scott, 05

Snodgrass, Stephen, OF

Speckmaier, Max, OF

Sullivan, Joseph F., 05, OC, OD, OE, ON

Te Plate, Maurice, 05, OD, OF

Telfer, Randal C., 05, 06, OC, OD, OE

Tournois, Severine C., OE

Trabert, M., Ol

Tuell, Michael T., OJ

Uhlendorf, K., OL

Vila, M. Begoña, 05

Voelkel, Reinhard, OA

Vogler, Uwe, OA

von Handorf, Robert J., OE

Ward, Justin, OF

Warner, Gerry D., 05

Wells, Martyn, OD

Wenzel, Greg W., 08, 0C

Werschnik, J., OL

Wolf, Erin M., OE

Wright, David, 05

Wright, Raymond H., 05

Young, Jerrod L., 08

Zhou, Julia, 05

Zielinski, Thomas P., 05, 06, OC

Proc. of SPIE Vol. $9951995101-6$ 


\title{
Conference Committee
}

\author{
Program Track Chairs
}

José Sassián, College of Optical Sciences, The University of Arizona (United States)

R. John Koshel, College of Optical Sciences, The University of Arizona (United States)

\section{Conference Chairs}

José Sasián, College of Optical Sciences, The University of Arizona (United States)

Richard N. Youngworth, Riyo LLC (United States)

Conference Program Committee

Matthew B. Dubin, College of Optical Sciences, The University of Arizona (United States)

Jonathan D. Ellis, University of Rochester (United States)

Sen Han, University of Shanghai for Science and Technology (China)

Marco Hanft, Carl Zeiss AG (Germany)

Chao-Wen Liang, National Central University (Taiwan)

Norbert Lindlein, Friedrich-Alexander-Universität Erlangen-Nürnberg (Germany)

Robert M. Malone, National Security Technologies, LLC (United States)

Raymond G. OhI IV, NASA Goddard Space Flight Center

(United States)

Craig W. Pansing, Synopsys, Inc. (United States)

Robert E. Parks, Optical Perspectives Group, LLC (United States)

Brian C. Primeau, Ball Aerospace \& Technologies Corporation (United States)

Martha Rosete-Aguilar, Universidad Nacional Autónoma de México (Mexico)

Peng Su, College of Optical Sciences, The University of Arizona (United States)

Yana Z. Williams, Atlas Material Testing Technology (United States) 
Session Chairs

1 Space Systems and JWST Alignment and Integration I

Robert M. Malone, National Security Technologies, LLC (United States)

2 Alignment Methods and Algorithms

Raymond G. OhI IV, NASA Goddard Space Flight Center

(United States)

3 Space Systems and JWST Alignment and Integration II

Brian C. Primeau, Ball Aerospace \& Technologies Corporation (United States)

4 Aspheres, Tolerancing, and Verification

Chao-Wen Liang, National Central University (Taiwan) 


\section{Introduction}

In this year of 2016 the Optical Systems Alignment, Tolerancing, and Verification $X$ conference celebrated its tenth anniversary! We all are delighted about the continued success of the conference. We thank the authors, audience, SPIE staff, and the conference committee for contributing to make the conference so successful and of interest to the optical engineering community.

The conference has been always held on Sunday and arranged in four oral presentation sessions, with poster sessions on Monday and Wednesday. Papers contributed to Space Systems and JWST Alignment and Integration I; Alignment Methods and Algorithms; Space Systems and JWST Alignment and Integration II; and Aspheres, Tolerancing, and Verification.

The talks about the JWST provided the audience with an update of the telescope and integration. These papers were insightful about the methodologies being used for the alignment and verification. In particular, instrument and pupil alignment issues were brought up as an important subject. We are especially grateful to Raymond $G$. Ohl for his role on attracting so many fine presentations about the JWST.

Three papers we like to highlight are "A toolbox of metrology-based techniques for optical system alignment," by Philip Coulter, et. al.; "Precision spacers as compensators," by Michael R. Kehoe, et. al.; and "The structure function as a metric for roughness and figure," by Robert Parks et. al.

Reflecting on the last 10 years we realize that the optical engineering community has indeed emphasized the topics championed by the conference: Alignment, Tolerancing, and Verification. Today it is unthinkable to embark into a major project without addressing each of these topics, and we are starting to see many specialists in these fields. We believe that the conference has contributed to the awareness and development of such fields.

We hope that the readers will enjoy and benefit from the collection of papers presented in this tenth volume. We look forward to another ten successful years to come, starting with our conference in 2017 at SPIE Optics and Photonics in San Diego.

José Sasián Richard N. Youngworth 
Proc. of SPIE Vol. $9951995101-10$

Downloaded From: https://www.spiedigitallibrary.org/conference-proceedings-of-spie on 26 Apr 2023 Terms of Use: https://www.spiedigitallibrary.org/terms-of-use 


\title{
The Advanced LIGO Detectors in the Era of First Discoveries
}

\author{
Daniel Sigg ${ }^{\mathrm{a}}$, \\ for the LIGO Scientific Collaboration ${ }^{\mathrm{b}}$, and the VIRGO Collaboration ${ }^{\mathrm{c}}$ \\ ${ }^{a}$ LIGO Hanford Observatory, 127124 North Route 10, Richland, WA 99354, USA \\ ${ }^{\mathrm{b}}$ web: www.ligo.org \\ ${ }^{c}$ web: www.virgo-gw.eu
}

\begin{abstract}
Following a major upgrade, the two advanced detectors of the Laser Interferometer Gravitational-wave Observatory (LIGO) held their first observation run between September 2015 and January 2016. The product of observable volume and measurement time exceeded that of all previous runs within the first 16 days of coincident observation. On September 14th, 2015 the Advanced LIGO detectors observed the transient gravitational-wave signal GW150914, determined to be the coalescence of two black holes, launching the era of gravitational-wave astronomy. We present the main features of the detectors that enabled this observation. At its core Advanced LIGO is a multi-kilometer long Michelson interferometer employing optical resonators to enhance its sensitivity. Four very pure and homogeneous fused silica optics with excellent figure quality serve as the test masses. The displacement produced by the event GW150914 was one 200th of a proton radius. It was observed with a combined signal-to-noise ratio of 24 in coincidence by the two detectors. At full sensitivity, the Advanced LIGO detectors are designed to deliver another factor of three improvement in the signal-to-noise ratio for binary black hole systems similar in masses to GW150914.
\end{abstract}

Keywords: LIGO, gravitational waves, black holes

\section{INTRODUCTION}

Einstein's theory of relativity predicts the existence of gravitational waves which are ripples in the space-time fabric and propagate at the speed of light. ${ }^{1,2}$ An indirect proof of their existence was first observed in the double pulsar system system PSR B1913+16 by Hulse, Taylor and Weisberg. ${ }^{3,4}$ On September 14th, 2015, both Advanced LIGO detectors in the USA, H1 in Hanford, Washington and L1 in Livingston, Lousiana, made the first direct measurement of gravitational waves. ${ }^{5}$ The event, GW150914, was the merger of two black holes, with masses of $36_{-4}^{+5} M_{\odot}$ and $29_{-4}^{+4} M_{\odot}$, into a black hole of approximately $62_{-4}^{+4} M_{\odot}(90 \%$ confidence level $){ }^{6}$ The equivalent of 3.0 solar masses of energy $\left(\simeq 5.4 \times 10^{47} \mathrm{~J}\right)$ was radiated in gravitational waves. The gravitational waves from this event, which occurred at a distance of $\simeq 410_{-180}^{+160} \mathrm{Mpc} \simeq 1.3 \times 10^{9}$ light years, changed the separation between the test masses by $\simeq 4 \times 10^{-18} \mathrm{~m}$, or about one 200 -th of a proton radius.

The Advanced LIGO detectors are $4 \mathrm{~km}$ long Michelson interferometers enhanced by multiple optical cavities. ${ }^{7,8}$ Each Michelson arm employs two test masses that form the arm cavities. Advanced LIGO came online in September 2015, after a major upgrade targeting a factor of 10 sensitivity improvement over initial detectors..$^{9,10}$ While not yet at design sensitivity during their first observation run, they have already exceeded the strain sensitivity of the initial detectors across the entire frequency band. This has significantly increased the discovery potential and led to the discovery of the first black hole merger. ${ }^{11-15}$

\section{THE ADVANCED LIGO DETECTORS}

The LIGO laboratory runs two observatories which house one Advance LIGO detector each. Figure 1 shows an areal view of the LIGO Hanford Observatory. The second observatory in Livingston is identical. Two or more observatories are required for coincident detection and to distinguish between signals of astrophysical origin and environmental disturbances.

Further author information:

E-mail: sigg_d@ligo.caltech.edu, Telephone: 15093728132

Interferometry XVIII, edited by Katherine Creath, Jan Burke, Armando Albertazzi Gonçalves Jr., Proc. of SPIE Vol. 9960, 996009 - (c) 2016 SPIE · CCC code: 0277-786X/16/\$18 · doi: 10.1117/12.2243115 


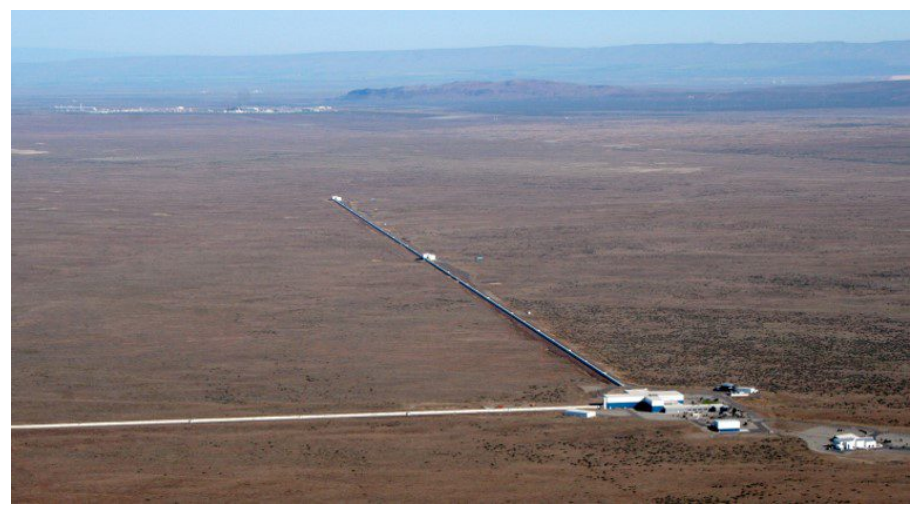

Figure 1. LIGO Hanford Observatory. The LIGO Hanford Observatory is located in eastern Washington. It houses one of the $4 \mathrm{~km}$ long Advanced LIGO detector.

The Advanced LIGO detectors are major upgrades to the initial detectors with much improved seismic isolation, quadruple pendulums, monolithic suspensions and electrostatic drives. The available laser power was also increased by a factor of 10 , but for the first run only $20-25 \mathrm{~W}$ were used. Installation of the new detectors finished in June 2014 in Livingston and in January 2015 in Hanford. Figure 2 shows the progress in commissioning between end of installation and the begin of the first observational run. The displayed range represents the skyaveraged reach to detect the coalescence of a binary neutron with a signal-to-noise ratio of 8 . The units are Mpc. These detectors, with a range of $70 \mathrm{Mpc}$, reach far beyond the local galaxy group and encompasses the entire Virgo supercluster. We also note that for heavier sources such as $30 M_{\odot}$ black hole mergers the range reaches beyond 1 GPc.

Figure 3 shows a simplified optical layout of the Advanced LIGO detector. A $200 \mathrm{~W}$ pre-stabilized laser (left) is used as the main light source at a wavelength of $1064 \mathrm{~nm}$ (red beams). ${ }^{16}$ An electro-optic modulator (not shown) is used to impose phase modulated RF sidebands at $9 \mathrm{MHz}$ (brown beams) and $45 \mathrm{MHz}$ (blue beams). It is spatially cleaned by a suspended input mode cleaner (not shown), ${ }^{17}$ before being injected into the dual-recycled Michelson interferometer with arm cavities. ${ }^{18}$ Each arm cavity comprises an input test mass (ITMX and ITMY) and an end test mass (ETMX and ETMY). The beamsplitter (BS) separates the main beam into the X-arm and

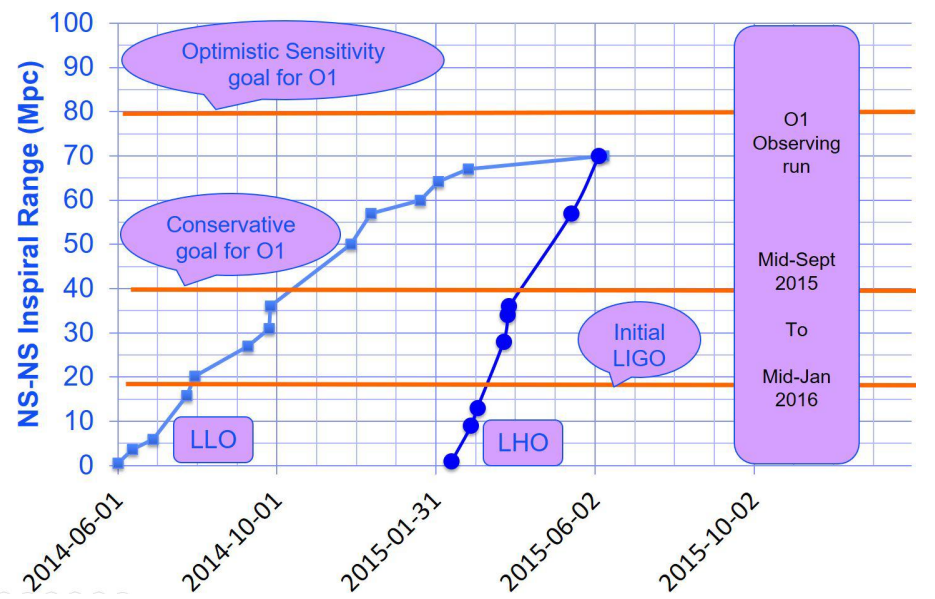

Figure 2. Commissioning progress. This graph shows the increase in binary neutron star reach as function of commissioning time, starting immediately after installation finished. The initial LIGO reach was surpassed within 2 months. The Hanford detector (LHO) finished installation about 8 months after the Livingston one (LLO), but caught up fast. Both detectors reached a sky-averaged range around $70 \mathrm{Mpc}$ for binary neutron star inspirals with a single detector SNR of 8 . 


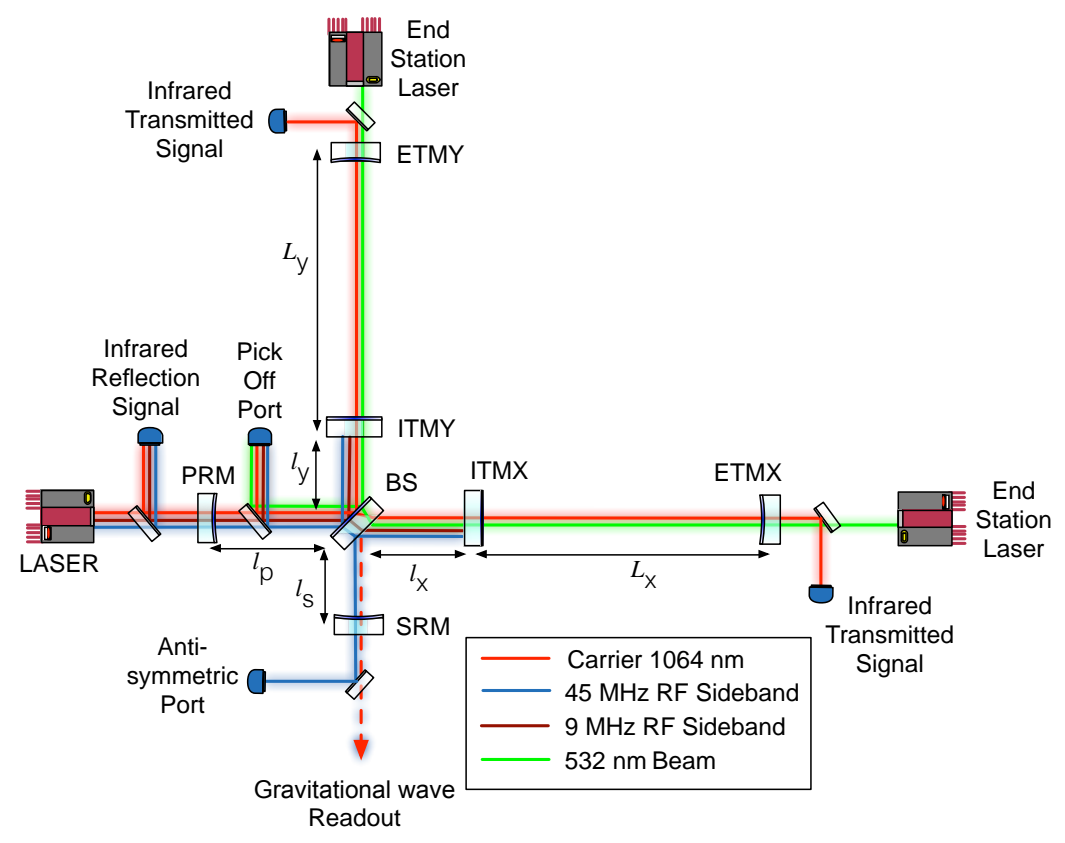

Figure 3. Simplified optical layout. See text for details.

the Y-arm. Two coupled cavities are formed around the Michelson vertex by the power recycling mirror (PRM) and by the signal recycling mirror (SRM), comprising the dual recycling configuration. ${ }^{19-22}$ Photodetectors are mounted in transmission of the arm cavities, at the antisymmetric port, in reflection of the interferometer and at the power recycling pick-off. Each of the multiple coupled optical cavities need to be locked to an exact multiple of the laser wavelength for operations. ${ }^{23}$ Furthermore, the beamsplitter is placed at a dark fringe. An $\mathrm{RF}$ scheme is used to sense most of the length and angular degrees-of-freedom. ${ }^{24,25}$ An output mode cleaner (not shown) is mounted at the anti-symmetric port to suppress unwanted light frequencies. The photodetectors in transmission of the output mode cleaner are used for the gravitational wave readout. The gravitational wave readout is sensed using a DC scheme by shifting the arm cavities slightly off resonance. ${ }^{26,27}$ Doubled Nd:YAG lasers running at a wavelength of $532 \mathrm{~nm}$ are installed behind the end test masses (green beams) and are used to lock the arm cavities independent of the main laser source ${ }^{23}$ but only during the lock acquisition process and not during observations.

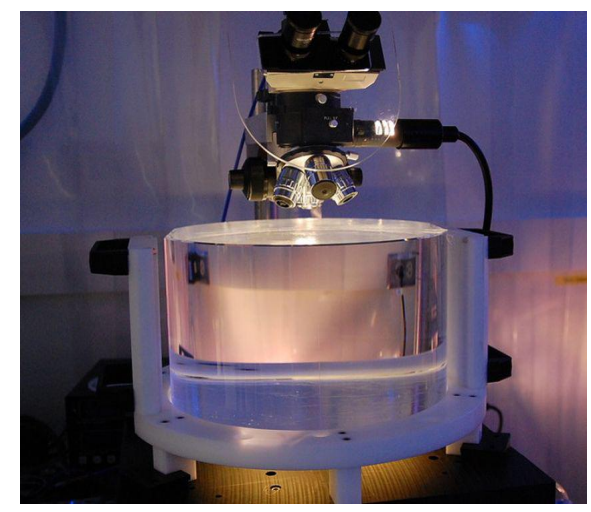

Figure 4. Test mass. 
High power operation poses special challenges with thermal lensing, angular instabilities driven by radiation pressure $^{28}$ and parametric instabilities. ${ }^{29}$ The system is typically locked at a lower power of $2 \mathrm{~W}$, where these effects are negligible, and then powered up. Thermal lenses are corrected with a thermal compensation system ${ }^{30}$ which comprises two CO2 lasers for central heating and four ring heaters for annular heating of the test masses. Parametric instabilities are driven by radiation pressure and the interaction between the test mass acoustic modes and the higher order optical modes. They are suppressed using active controls which applies damping filters. This system needs to engage during the power up to prevent run-away oscillations which break the lock. The higher the optical power in the interferometer the larger number of parametric instabilities are present. This makes the problem relatively easy at $20 \mathrm{~W}$ but hard at $200 \mathrm{~W}$. Angular instabilities due to radiation pressure also depend on the power in the interferometer and become harder to damp at higher power. The current strategy requires angular servos with a higher bandwidth than the frequencies of the angular instabilities. However, increasing the bandwidth of the angular servos introduces additional noise in the gravitational wave readout, unless there is a sharp cut-off filter in the response.

A picture of a test mass in the metrology lab is shown in Figure 4. The Advanced LIGO test masses are very pure and homogeneous fused silica mirrors of $34 \mathrm{~cm}$ diameter, $20 \mathrm{~cm}$ thickness and $40 \mathrm{~kg}$ mass. It is critical that the test masses be free from sources of displacement noise, such as environmental disturbances from seismic noise ${ }^{31}$ or thermally driven motion. To reduce the effects of ground vibrations, the test masses are suspended by multi-stage pendulums, ${ }^{32}$ thus acting as free masses well above the lowest pendulum resonance frequency of $0.4 \mathrm{~Hz}$. Monolithic fused silica fibers ${ }^{33}$ are incorporated at the bottom stage to decrease suspension thermal noise ${ }^{34}$ which limits the sensitive band.. The Advanced LIGO test masses require about 10 orders of magnitude suppression of ground motion above $10 \mathrm{~Hz}$. The multi-stage pendulum system attenuates the ground motion by seven orders of magnitude. It is mounted on an actively controlled seismic isolation platform which provides three orders of magnitude of isolation of its own. ${ }^{35,36}$ Moreover, these platforms are used to reduce the very large displacements produced by tidal motion and microseismic activity. Tidal forces can produce displacements up to several $100 \mu \mathrm{m}$ over a multi-kilometer baseline on time scales of hours. The dominant microseismic activity is driven by ocean waves. The resulting ground motion can be as large as several $\mu \mathrm{m}$ at frequencies around $0.15 \mathrm{~Hz}$ - even far inland.

The entire test mass assembly including the suspension system and part of the seismic isolation system resides inside an ultra-high vacuum system, with pressures typically below $1 \mu \mathrm{Pa}$ over the $10,000 \mathrm{~m}^{3}$ volume, to prevent acoustic shorting of the seismic isolation systems and to reduce Rayleigh scattering in the optical readout.
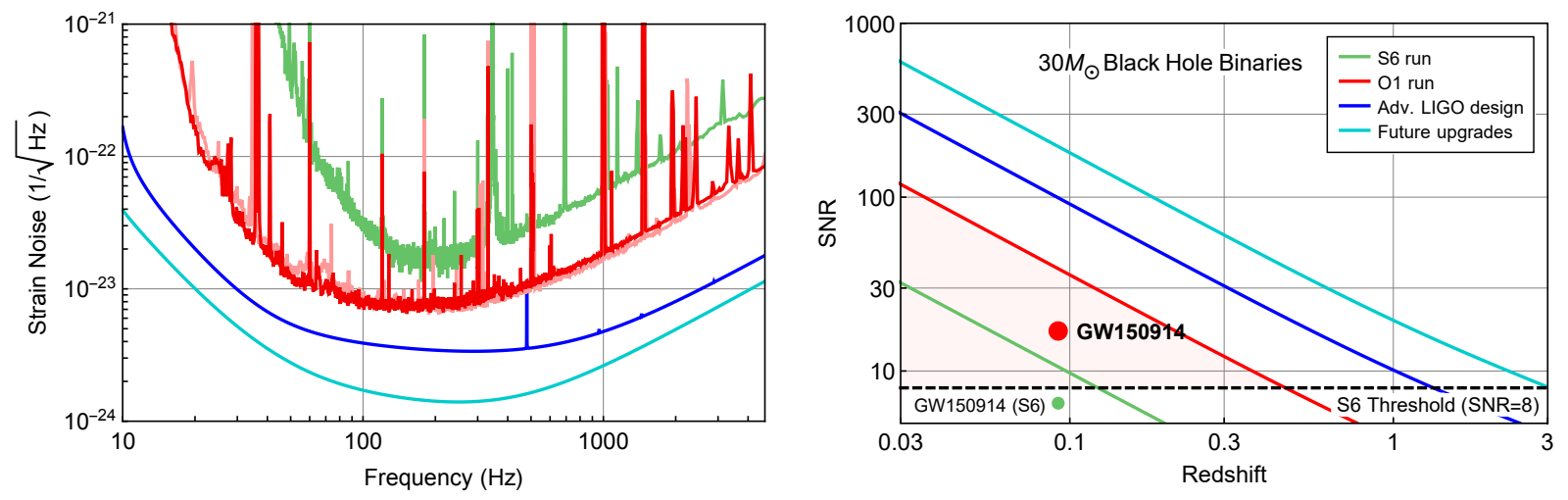

Figure 5. Sensitivity during the first observation run. ${ }^{7}$ The left plot shows the strain sensitivity during the first observation run (O1) of the Advanced LIGO detectors and during the last science run (S6) of the initial LIGO detectors. The O1 strain noise curve is shown for H1 (dark red) and L1 (light red); the two detectors have similar performance. The Advanced LIGO design sensitivity as well as a possible future upgrade ${ }^{37}$ are shown to highlight the discovery potential in the coming years. The right plot shows the single detector signal-to-noise ratio (SNR) under optimal orientation as function of redshift $z$ - for two merging black holes with mass $30 M_{\odot}$ each. GW150914 was not optimally orientated and was detected with a single detector SNR of 13 to 20 at $z=0.09$; this event would not have been detected in initial LIGO. 


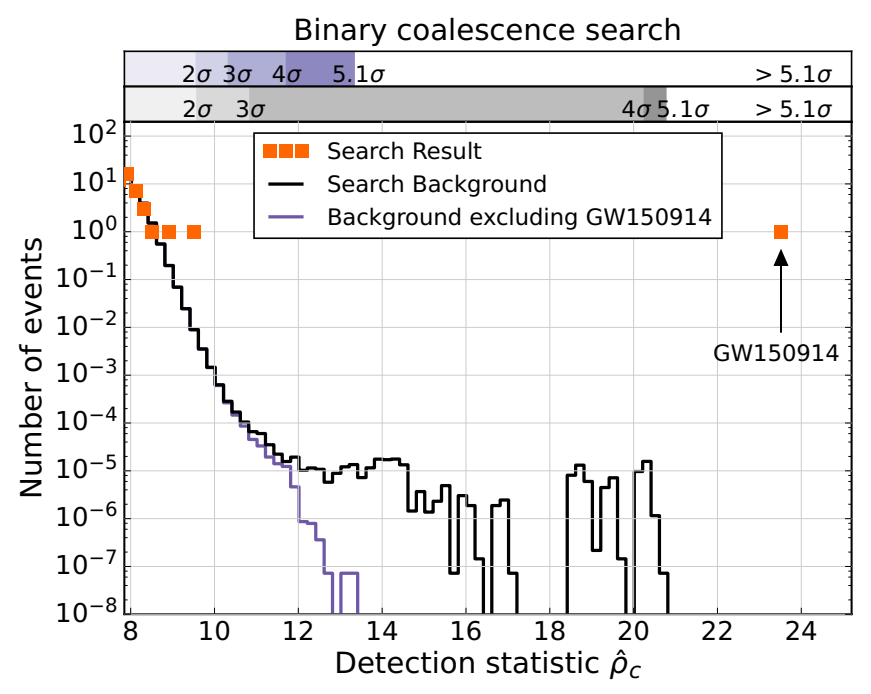

Figure 6. First detection. ${ }^{5}$ The foreground events are denoted by the red squares. The background is estimated using time shifted data from the two detectors. GW150914 is the most significant event in the first 16 days of coincident observation. When the event is removed form the data (blue line), the remaining background is clean. If we look at the search background including GW150914, we see a tail towards higher detection statistics which consist of the event being detected in one of the detectors in coincidence with a slightly elevated background in the other detector.

\section{THE FIRST DETECTION}

The sensitivity of the Advanced LIGO detectors during the first observation run $^{38}$ is shown in Figure 5. The sensitivity is significantly better than what was achieved during the last science run of the initial detectors. This is especially true for frequencies below $200 \mathrm{~Hz}$ which are most important for black hole mergers. It is important to remember that the observed volume and thus the number of possible sources scales with the third power of the sensitivity. Reaching the Advanced LIGO design sensitivity will be crucial in making black hole mergers a routine occurrence in astrophysical observations.

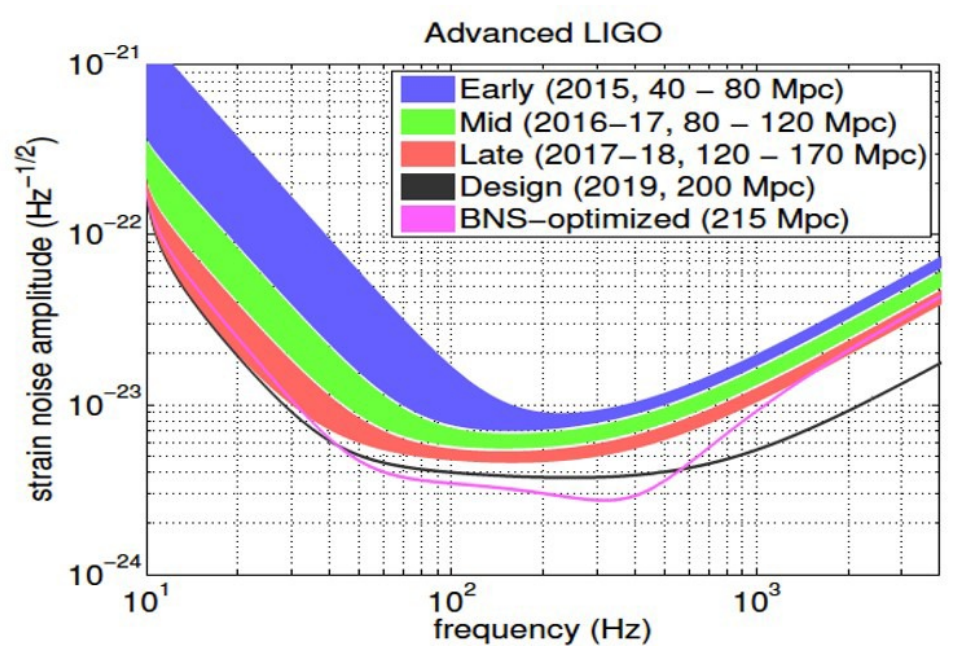

Figure 7. Planned observation periods. ${ }^{39}$ The first observation run is depicted by the blue band. The second observation run is depicted by the green band and will take place in the second half of 2016 and early 2017. A third observation run is currently being planned for the late 2017 and 2018 time frame. 
The first black hole merger event GW150914 was observed with a very high confidence level, see Figure 6 . The event was detected by both LIGO observatories in coincident. It also constitutes the single largest such event in either of the two detectors. We estimate the background rate by analysing the time shifted data streams between the two observatories.

\section{FUTURE PLANS}

Future plans are depicted in Figure 7. With the successful completion of the first observation run, the commissioning for the second observation run is in progress. The second observation run is currently being planned to start in September 2016.

The second observation run, LIGO will be joined by the Virgo detector ${ }^{40}$ which is located near Pisa, Italy. Having a third detector in the network is important for sky localization. With the LIGO detectors alone, the sky localization $90 \%$ confidence region can encompass several 100 square degrees. With the addition of the Virgo detector this potentially can be reduced to several square degrees.

The currently operating detectors are LIGO Hanford and LIGO Livingston as well as the GEO600 ${ }^{41}$ detector. However, GEO600 is not sensitive enough to detect black hole mergers. The Virgo detector and the Japanese KAGRA detector ${ }^{42}$ are under construction and will come online over the next year or two. The third Advanced LIGO detector is planned to be installed in India. ${ }^{43}$ Once the global network is active, we will be able to achieve good localization on any point in the sky as well as provide good coverage.

\section{CONCLUSIONS}

The LIGO Scientific Collaboration and the Virgo Collaboration have made the first detection of a black hole merger. This constitutes the first direct detection of gravitational waves on Earth. This is the beginning of the era of gravitational wave astrophysics with the promise of many more detections in the near future.

\section{ACKNOWLEDGMENTS}

The authors gratefully acknowledge the support of the United States National Science Foundation (NSF) for the construction and operation of the LIGO Laboratory and Advanced LIGO as well as the Science and Technology Facilities Council (STFC) of the United Kingdom, the Max-Planck-Society (MPS), and the State of Niedersachsen/Germany for support of the construction of Advanced LIGO and construction and operation of the GEO600 detector. Additional support for Advanced LIGO was provided by the Australian Research Council. The authors gratefully acknowledge the Italian Istituto Nazionale di Fisica Nucleare (INFN), the French Centre National de

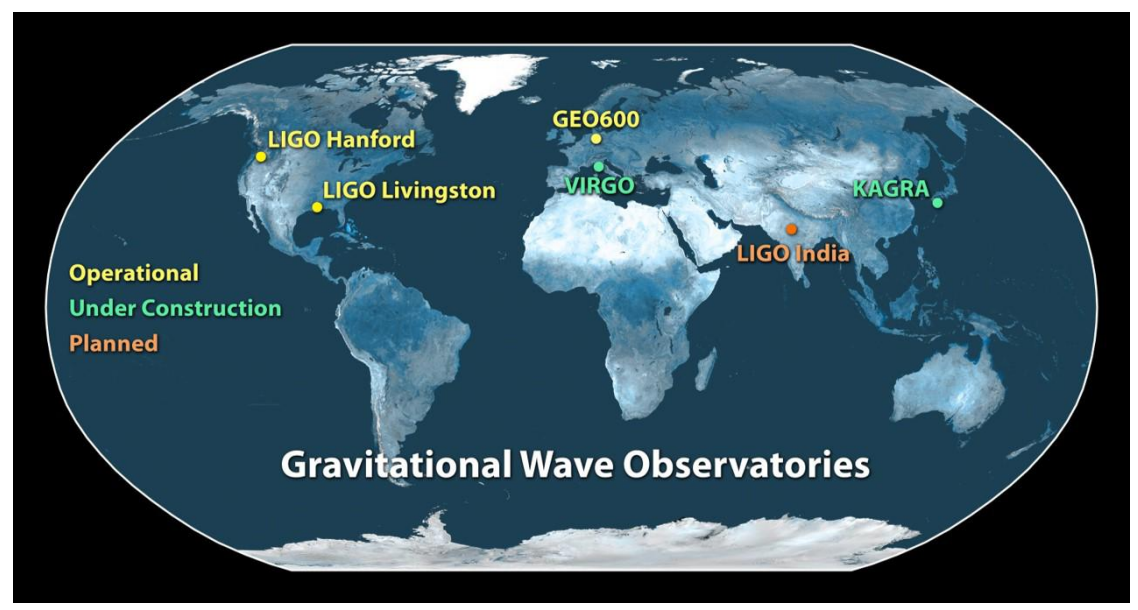

Figure 8. Detector network. This world map depicts the location of the planned network of ground based gravitational wave antennas. 
la Recherche Scientifique (CNRS) and the Foundation for Fundamental Research on Matter supported by the Netherlands Organisation for Scientific Research, for the construction and operation of the Virgo detector and the creation and support of the EGO consortium. The authors also gratefully acknowledge research support from these agencies as well as by the Council of Scientific and Industrial Research of India, Department of Science and Technology, India, Science \& Engineering Research Board (SERB), India, Ministry of Human Resource Development, India, the Spanish Ministerio de Economía y Competitividad, the Conselleria d'Economia i Competitivitat and Conselleria d'Educació, Cultura i Universitats of the Govern de les Illes Balears, the National Science Centre of Poland, the European Commission, the Royal Society, the Scottish Funding Council, the Scottish Universities Physics Alliance, the Hungarian Scientific Research Fund (OTKA), the Lyon Institute of Origins (LIO), the National Research Foundation of Korea, Industry Canada and the Province of Ontario through the Ministry of Economic Development and Innovation, the Natural Science and Engineering Research Council Canada, Canadian Institute for Advanced Research, the Brazilian Ministry of Science, Technology, and Innovation, Fundação de Amparo à Pesquisa do Estado de São Paulo (FAPESP), Russian Foundation for Basic Research, the Leverhulme Trust, the Research Corporation, Ministry of Science and Technology (MOST), Taiwan and the Kavli Foundation. The authors gratefully acknowledge the support of the NSF, STFC, MPS, INFN, CNRS and the State of Niedersachsen/Germany for provision of computational resources.

This document has been assigned the LIGO Laboratory document number LIGO-P1600182.

\section{REFERENCES}

[1] Einstein, A., "Näherungsweise Integration der Feldgleichungen der Gravitation," Sitzungsber. K. Preuss. Akad. Wiss. 1, 688 (1916).

[2] Einstein, A., "Gravitationswellen," Sitzungsber. K. Preuss. Akad. Wiss. 1, 154 (1918).

[3] Hulse, R. A. and Taylor, J. H., "Discovery of a pulsar in a binary system," Astrophys. J. 195, L51 (1975).

[4] Taylor, J. H. and Weisberg, J. M., "A new test of general relativity - Gravitational radiation and the binary pulsar PSR 1913+16," Astrophys. J. 253, 908 (1982).

[5] Abbott, B. P. et al. (LIGO Scientific Collaboration and Virgo Collaboration), "Observation of gravitational waves from a binary black hole merger," Phys. Rev. Lett. 116, 061102 (2016).

[6] Abbott, B. P. et al. (LIGO Scientific Collaboration and Virgo Collaboration), "Properties of the binary black hole merger GW150914," Phys. Rev. Lett. 116, 241102 (2016).

[7] Abbott, B. P. et al. (LIGO Scientific Collaboration and Virgo Collaboration), "GW150914: The Advanced LIGO detectors in the era of first discoveries," Phys. Rev. Lett. 116, 131103 (2016).

[8] Aasi, J. et al., "Advanced LIGO," Class. Quantum Grav. 32(7), 074001 (2015).

[9] Abbott, B. P. et al., "LIGO: the Laser Interferometer Gravitational-wave Observatory," Rep. Prog. Phys. 72(7), 076901 (2009).

[10] Accadia, T. et al., "Virgo: a laser interferometer to detect gravitational waves," J. Inst. 7(03), P03012 (2012).

[11] Abbott, B. P. et al. (LIGO Scientific Collaboration and Virgo Collaboration), "Astrophysical implications of the binary black hole merger GW150914," Astrophys. J. Lett. 818(2), L22 (2016).

[12] Abbott, B. P. et al. (LIGO Scientific Collaboration and Virgo Collaboration), "Tests of general relativity with GW150914," Phys. Rev. Lett. 116, 221101 (2016).

[13] Abbott, B. P. et al. (LIGO Scientific Collaboration and Virgo Collaboration), "GW150914: Implications for the stochastic gravitational-wave background from binary black holes," Phys. Rev. Lett. 116, 131102 (2016).

[14] Abbott, B. P. et al. (LIGO Scientific Collaboration, Virgo Collaboration, Australian Square Kilometer Array Pathfinder (ASKAP) Collaboration, BOOTES Collaboration, Dark Energy Survey and the Dark Energy Camera GW-EM Collaborations, Fermi GBM Collaboration, Fermi LAT Collaboration, GRAvitational Wave Inaf TeAm (GRAWITA), INTEGRAL Collaboration, Intermediate Palomar Transient Factory (iPTF) Collaboration, The InterPlanetary Network, J-GEM Collaboration, La Silla-QUEST Survey, Liverpool Telescope Collaboration, Low Frequency Array (LOFAR) Collaboration, MASTER Collaboration, MAXI Collaboration, Murchison Wide-field Array (MWA) Collaboration, Pan-STARRS Collaboration, PESSTO Collaboration, Pi of the Sky Collaboration, SkyMapper Collaboration, Swift Collaboration, TAROT, Zadko, 
Algerian National Observatory, and C2PU Collaboration, TOROS Collaboration and VISTA Collaboration), "Localization and broadband follow-up of the gravitational-wave transient GW150914," Astrophys. J. Lett. 0, in press (2016).

[15] Adrián-Martínez, S. et al. (ANTARES Collaboration, IceCube Collaboration, LIGO Scientific Collaboration and Virgo Collaboration), "High-energy neutrino follow-up search of gravitational wave event GW150914 with ANTARES and IceCube," Phys. Rev. D 93, 122010 (2016).

[16] Kwee, P. et al., "Stabilized high-power laser system for the gravitational wave detector Advanced LIGO," Opt. Express 20, 10617-10634 (May 2012).

[17] Mueller, C. L. et al., "The Advanced LIGO input optics," Rev. Sci. Instrum. 87(1), 014502 (2016).

[18] Drever, R. W. P., [Fabry-Perot cavity gravity wave detectors], Cambridge University Press (July 1991).

[19] Drever, R. W. P. et al., "Quantum optics," in [Quantum Optics, Experimental Gravity, and Measurement Theory], Meystre, P. and Scully, M. O., eds., NATO ASI Series B 94, 503-514, Plenum Press, New York (1983).

[20] Schilling, R. (personal communication).

[21] Meers, B. J., "Recycling in laser-interferometric gravitational-wave detectors," Phys. Rev. D 38, 2317-2326 (October 1988).

[22] Mizuno, J. et al., "Resonant sideband extraction: a new configuration for interferometric gravitational wave detectors," Phys. Lett. A 175(5), 273-276 (1993).

[23] Staley, A., Martynov, D., et al., "Achieving resonance in the Advanced LIGO gravitational-wave interferometer," Class. Quantum Grav. 31(24), 245010 (2014).

[24] Fritschel, P. et al., "Readout and control of a power-recycled interferometric gravitational-wave antenna," Appl. Opt. 40, 4988-4998 (Oct 2001).

[25] Barsotti, L., Evans, M., and Fritschel, P., "Alignment sensing and control in Advanced LIGO," Class. Quantum Grav. 27(8), 084026 (2010).

[26] Fricke, T. T. et al., "DC readout experiment in Enhanced LIGO," Class. Quantum Grav. 29(6), 065005 (2012).

[27] Hild, S. et al., "DC-readout of a signal-recycled gravitational wave detector," Class. Quantum Grav. 26(5), 055012 (2009).

[28] Sidles, J. A. and Sigg, D., "Optical torques in suspended Fabry-Perot interferometers," Phys. Lett. A 354(3), 167-172 (2006).

[29] Evans, M. et al., "Observation of parametric instability in Advanced LIGO," Phys. Rev. Lett. 114, 161102 (Apr 2015).

[30] Brooks, A. F. et al., "Direct measurement of absorption-induced wavefront distortion in high optical power systems," Appl. Opt. 48, 355-364 (Jan 2009).

[31] Effler, A. et al., "Environmental influences on the LIGO gravitational wave detectors during the 6th science run," Class. Quantum Grav. 32(3), 035017 (2015).

[32] Aston, S. M. et al., "Update on quadruple suspension design for Advanced LIGO," Class. Quantum Grav. 29(23), 235004 (2012).

[33] Braginskii, V. B., Mitrofanov, V. P., and Okhrimenko, O. A., "Oscillators for free-mass gravitational antennas," Sov. J. Exp. Theor. Phys. Lett. 55, 432 (Apr 1992).

[34] Cumming, A. V. et al., "Design and development of the advanced LIGO monolithic fused silica suspension," Class. Quantum Grav. 29(3), 035003 (2012).

[35] Matichard, F. et al., "Seismic isolation of Advanced LIGO: review of strategy, instrumentation and performance," Class. Quantum Grav. 32(18), 185003 (2015).

[36] Wen, S. et al., "Hydraulic external pre-isolator system for LIGO," Class. Quantum Grav. 31(23), 235001 (2014).

[37] Miller, J., Barsotti, L., Vitale, S., Fritschel, P., Evans, M., and Sigg, D., "Prospects for doubling the range of Advanced LIGO," Phys. Rev. D 91, 062005 (Mar 2015).

[38] Martynov, D. V., Hall, E. D. et al. (LIGO Scientific Collaboration), "The sensitivity of the Advanced LIGO detectors at the beginning of gravitational wave astronomy," Phys. Rev. D 93, 112004 (2016).

xviii 
[39] Abbott, B. P. et al. (LIGO Scientific Collaboration and Virgo Collaboration), "Prospects for observing and localizing gravitational-wave transients with Advanced LIGO and Advanced Virgo," Liv. Rev. Relat. 19(1), 1 (2016).

[40] Acernese, F. et al., "Advanced Virgo: a second-generation interferometric gravitational wave detector," Class. Quantum Grav. 32(2), 024001 (2015).

[41] Affeldt, C., Danzmann, K., Dooley, K. L., Grote, H., Hewitson, M., Hild, S., Hough, J., Leong, J., Lck, H., Prijatelj, M., Rowan, S., Rdiger, A., Schilling, R., Schnabel, R., Schreiber, E., Sorazu, B., Strain, K. A., Vahlbruch, H., Willke, B., Winkler, W., and Wittel, H., "Advanced techniques in GEO 600," Class. Quantum Grav. 31(22), 224002 (2014).

[42] Aso, Y. et al., "Interferometer design of the KAGRA gravitational wave detector," Phys. Rev. D 88, 043007 (Aug 2013).

[43] Iyer, B. et al., "LIGO-India," Tech. Rep. LIGO-M1100296 (2011). 
Proc. of SPIE Vol. $9951995101-20$

Downloaded From: https://www.spiedigitallibrary.org/conference-proceedings-of-spie on 26 Apr 2023 Terms of Use: https://www.spiedigitallibrary.org/terms-of-use 